\title{
Corrigendum to "Nicotine Enhances Interspecies Relationship between Streptococcus mutans and Candida albicans"
}

\author{
Shiyu Liu, ${ }^{1,2}$ Wei Qiu, ${ }^{1}$ Keke Zhang, ${ }^{1}$ Xuedong Zhou, ${ }^{1,2}$ Biao Ren, ${ }^{1}$ Jinzhi He, ${ }^{1,2}$ \\ Xin $\mathrm{Xu}^{1,2}$ Lei Cheng, ${ }^{1,2}$ and Mingyun $\mathrm{Li}^{1}$ \\ ${ }^{1}$ State Key Laboratory of Oral Diseases, Sichuan University, Chengdu, China \\ ${ }^{2}$ Department of Operative Dentistry and Endodontics, West China Hospital of Stomatology, Sichuan University, Chengdu, China \\ Correspondence should be addressed to Lei Cheng; chenglei@scu.edu.cn and Mingyun Li; limingyun@scu.edu.cn
}

Received 26 July 2017; Accepted 24 August 2017; Published 20 September 2017

Copyright (C) 2017 Shiyu Liu et al. This is an open access article distributed under the Creative Commons Attribution License, which permits unrestricted use, distribution, and reproduction in any medium, provided the original work is properly cited.

In the article titled "Nicotine Enhances Interspecies Relationship between Streptococcus mutans and Candida albicans" [1], an acknowledgment should be added as follows.

This study was supported by the National Natural Science Foundation of China (81400501 to Mingyun Li and 81430011 to Xuedong Zhou), the International Science and Technology Cooperation Program of China (2014DFE30180 to Xuedong Zhou), and the Special Fund of State Key Laboratory of Oral Diseases, Sichuan University (SKLOD201525 to Mingyun Li).

\section{References}

[1] S. Liu, W. Qiu, K. Zhang et al., "Nicotine enhances interspecies relationship between streptococcus mutans and candida albicans," BioMed Research International, vol. 2017, Article ID 7953920, 9 pages, 2017. 

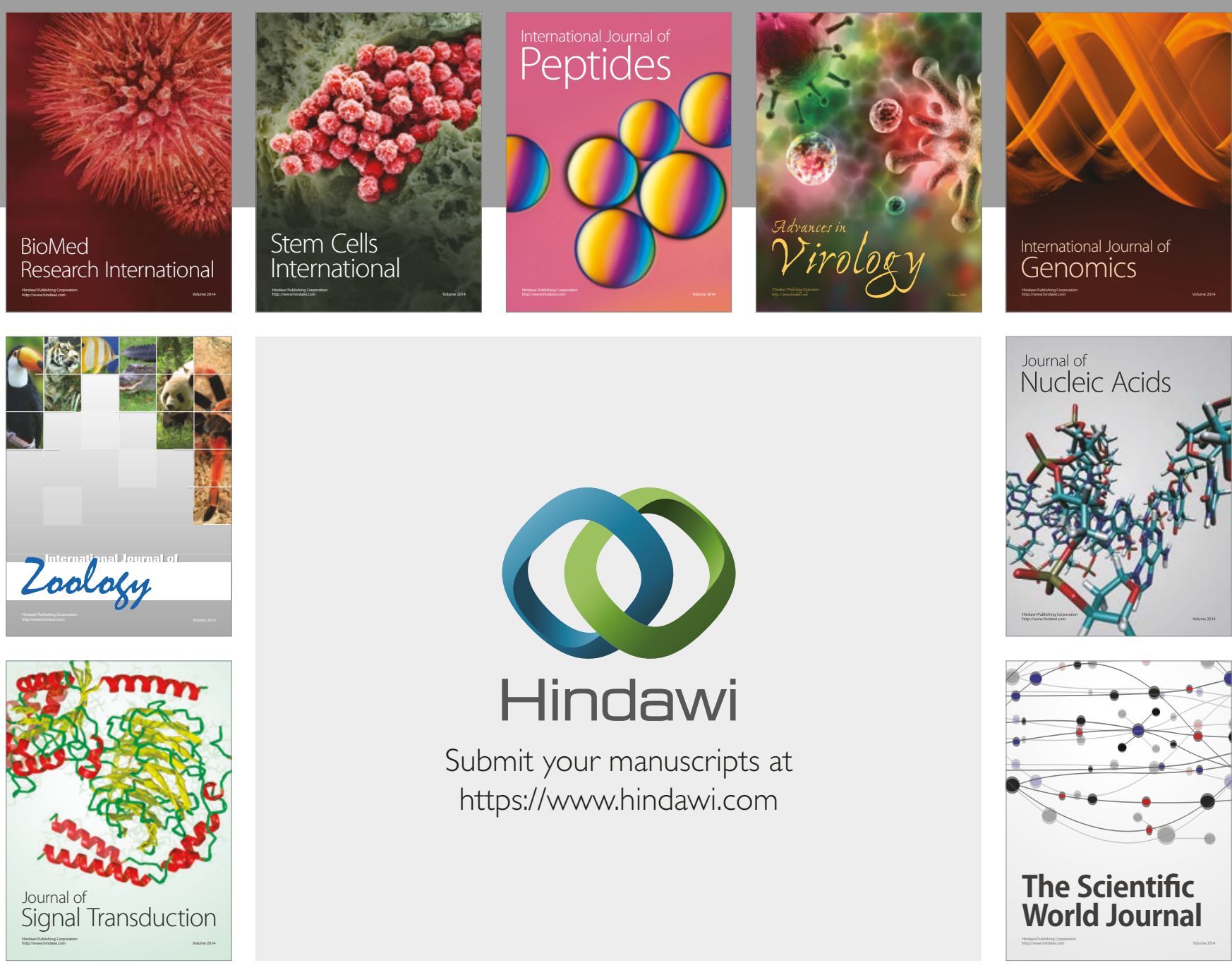

Submit your manuscripts at

https://www.hindawi.com
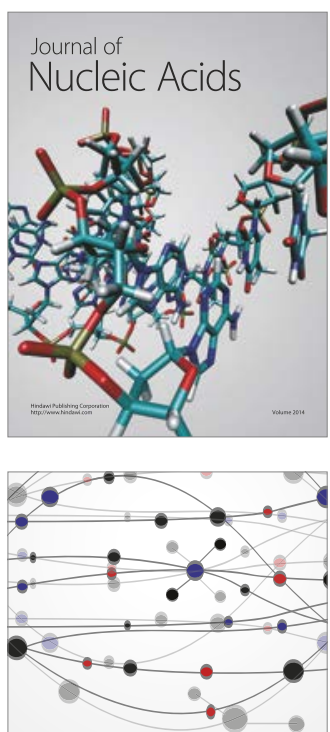

The Scientific World Journal

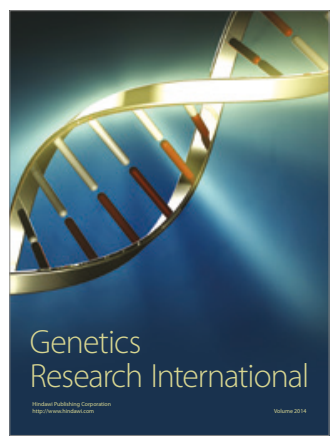

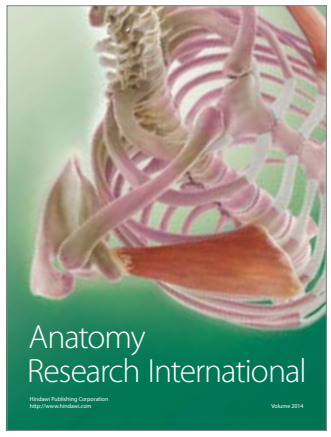

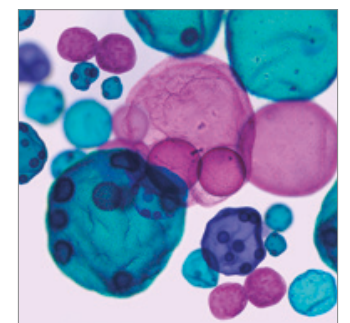

International Journal of Microbiology
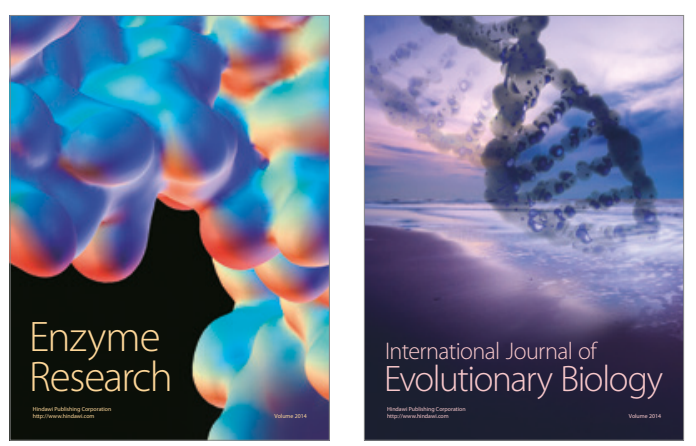
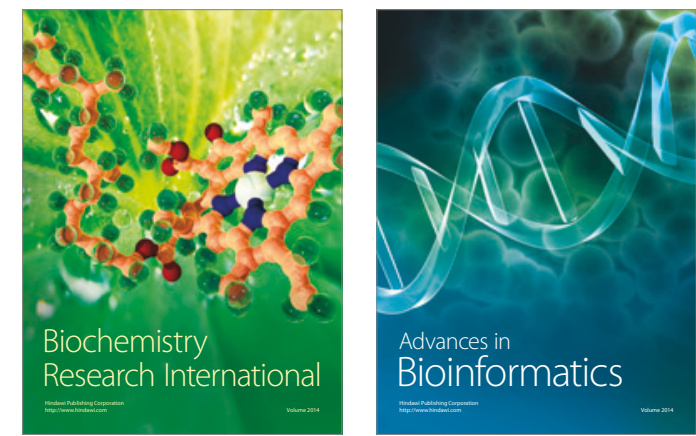

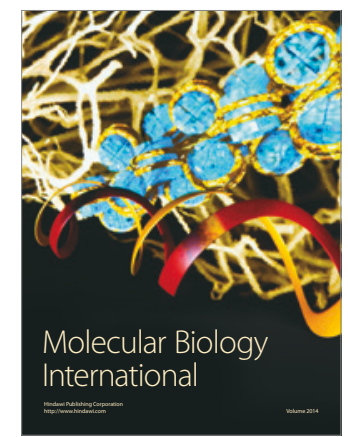

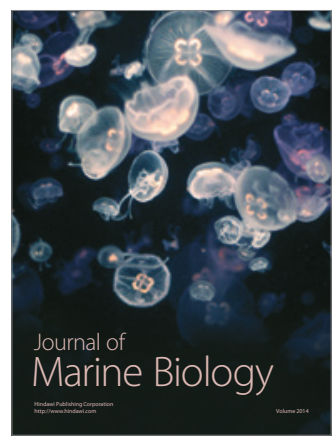

